\title{
TRANSPORT IN MAGNETIC LAYERED STRUCTURES: GIANT MAGNETORESISTANCE
}

\author{
J. BARNAŚ \\ Magnetism Theory Division, Institute of Physics, A. Mickiewicz University \\ Matejki 48/49, 60-769 Poznań, Poland
}

Basic features of electronic transport in magnetic layered structures consisting of alternating ferromagnetic and nonmagnetic metallic films are analysed theoretically. The considerations are restricted to magnetoresistance originating from the rotation of the film magnetizations from antiparallel to parallel alignment. The influence of spin-dependent potentials on the transport properties is analysed for both current-in-plane and current-perpendicular-to-plane geometries. Quasi-classical and quantum methods are used to calculate the appropriate conductiyity. For current-perpendicular-to-plane geometry the periodic spin-dependent effective electron potential plays an important role and can generate giant magnetoresistance also when the electron scattering by impurities and interface roughness is independent of the spin direction, contrary to the case of current-in-plane geometry when a spin-dependent scattering probability is a necessary condition for the giant magnetoresistance to occur.

PACS numbers: 75.70.Cn, 72.15.Gd, 75.50.Rr

\section{Introduction}

Owing to the discovery of giant magnetoresistance (GMR) in $\mathrm{Fe} / \mathrm{Cr}$ layered structures $[1,2]$ transport in magnetic layered structures attracted much attention in last few years. Recently, many details of the effect have been reported and the effect was found in many other layered systems containing ferromagnetic transition-metal sublayers [3]. The simplest structures in which GMR occurs consist of two ferromagnetic films, say of thickness $d_{m}$, separated by a nonmagnetic spacer of thickness $d_{\mathrm{n}}$ with antiparallel magnetizations in zero external magnetic field. The antiparallel alignment is usually obtained owing to antiferromagnetic interlayer exchange coupling which exists at some spacer thicknesses, but the existence of the coupling is not necessary for GMR to occur [4] and the antiparallel alignment can also be achieved by other means. The magnetizations rotate to parallel alignment in appropriate external field and the GMR consists in a large change (drop or increase) of the resistance at the transition from the antiparallel to parallel alignment of the film magnetizations. 
The effect is usually described quantitatively by the ratio

$$
\frac{\Delta R}{R_{\uparrow \uparrow}}=\frac{R_{\uparrow \downarrow}-R_{\uparrow \uparrow}}{R_{\uparrow \uparrow}}
$$

with $R_{\uparrow \downarrow}$ and $R_{\uparrow \uparrow}$ being the resistances in antiparallel and parallel configuration, respectively. In all experimental data published up to now the ratio $\Delta R / R_{\uparrow \uparrow}$ was positive, which corresponds to negative magnetoresistance (MR), but a negative value of $\Delta R / R_{\uparrow \uparrow}$ is also allowed. In the following the effect will be called GMR or equivalently MR (GMR is simply a sufficiently large MR). The basic features of the effect are: (i) the effect increases with decreasing temperature, (ii) it increases with increasing number of magnetic films in the multilayer and (iii) it generally increases with decreasing sublayer thicknesses.

In most experiments the GMR was investigated for electric current flowing parallel to the film plane (current-in-plane (CIP) geometry), but recently two experimental groups have also succeeded to obtain GMR for currents flowing perpendicularly to the structure (current perpendicular-to-plane (CPP) geometry) $[5,6]$. In the latter case the effect is usually larger than in the CIP geometry.

It is generally accepted that spin-dependent transport in ferromagnetic transition metals is responsible for GMR. In general, one can distinguish two different contributions to the effect. One of them is due to spin-dependent scattering probability (described by some effective spin-dependent scattering potential $V_{\sigma}$ ) due to impurities inside the films (bulk scattering) and/or interface roughness (interface scattering). Another one comes from the periodic spin-dependent effective electron potential $U_{\sigma}$ of a pure and ideal structure (this spin-dependent potential is responsible for the spin-splitting of the electron bands in ferromagnetic metals). The scattering potential $V_{\sigma}$ contributes in both CIP and CPP configurations, whereas the potential $U_{\sigma}$ is effective only in the CPP geometry.

Recent experiments $[5,6]$ in the CPP configuration raised several additional fundamental questions. One of them is the role of spin accumulation layer at the interface between ferromagnetic and nonmagnetic metals and the role of spin-flip electron scattering.

Below we describe briefly basic methods of theoretical description of the effect as well as the most important results. We start with a short qualitative picture in the CIP geometry, which explains physics of the effect. Then, we describe the quasi-classical and quantum methods used for evaluation of the MR. Finally, we describe briefly the GMR in the CPP geometry.

\section{CIP geometry}

\subsection{Qualitative considerations}

Let us start with a qualitative description of the GMR. The effect can be accounted for within the two-current model developed by Campbell and Fert [7]. The model takes into account the fact that the electronic charge in transition ferromagnetic metals is carried via two non-equivalent spin-majority and spin-minority channels. At low temperatures spin-flip scattering processes are frozen out and the spin relaxation time $\tau_{\sigma}^{\text {sf }}$ is much larger than the momentum relaxation time $\tau_{\sigma}$. 
Consequently, the two parallel conduction channels can be considered as independent in the first approximation. They can also be assumed as independent when the sample size is much smaller than the spin-diffusion length. Each spin-channel can be then characterized by its own mean free path $\lambda_{\sigma}\left(\lambda_{\sigma}=v_{\sigma}^{\mathrm{F}} \tau_{\sigma}\right.$, where $v_{\sigma}^{\mathrm{F}}$ is the Fermi velocity which can be spin-dependent, in general). The GMR can be understood qualitatively by taking into account a spin-asymmetry in scattering probabilities.

Consider first the macroscopic limit, when the sublayer thicknesses are much larger than the mean free paths for both spin-majority $(+)$ and spin-minority $(-)$ electrons. The conduction electrons essentially do not sample then two sublayers between successive scattering events, therefore any bilayer can be replaced by two films without direct contact and, consequently, by two resistors connected in parallel. In the case of independent spin-channels the total resistance of the parallel and antiparallel configurations is equal to the resistance of the appropriate resistor network as shown schematically in Fig. 1 for a simple symmetrical sandwich. It is

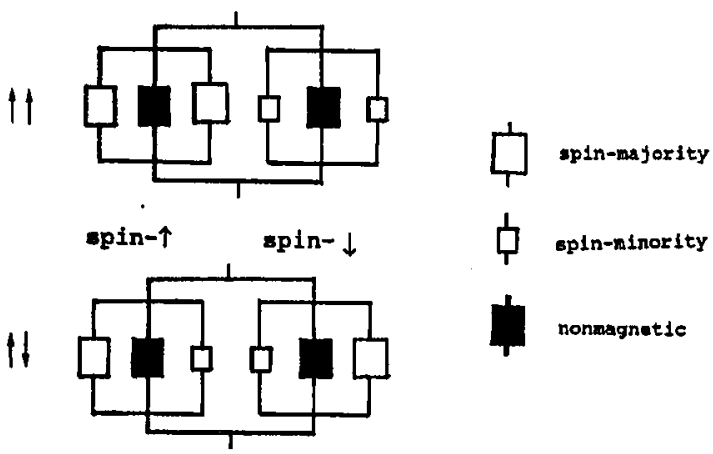

Fig. 1. Resistor networks for the parallel and antiparallel configurations valid in the macroscopic limit of the CIP geometry.

evident that the total resistances of both configurations are equal. Thus, there is no GMR in the macroscopic limit in the CIP geometry.

The above arguments are not valid if $\lambda_{+}$and/or $\lambda_{-}$are comparable to or smaller than the sublayer thicknesses. In that case the model shown in Fig. 1 is not valid. The question arises whether the spin-dependent scattering processes can lead to GMR in the CIP geometry or not. To solve the problem qualitatively let us consider a symmetrical sandwich with a large spin-asymmetry in probability of electron scattering from impurities located inside the magnetic films (described by the spin-asymmetry factor $N_{\mathrm{b}}=\tau_{-} / \tau_{+}$) and/or from interface roughness (described by the factor $N_{\mathrm{s}}$ ). It is easy to conclude from Fig. 2 that the spin-asymmetry in scattering probabilities leads to MR indeed. Only the case of bulk scattering is shown there but the same arguments also apply to interface scattering. In Fig. 2 the spin-minority electrons are assumed to be scattered very weakly ("fast electrons"), 

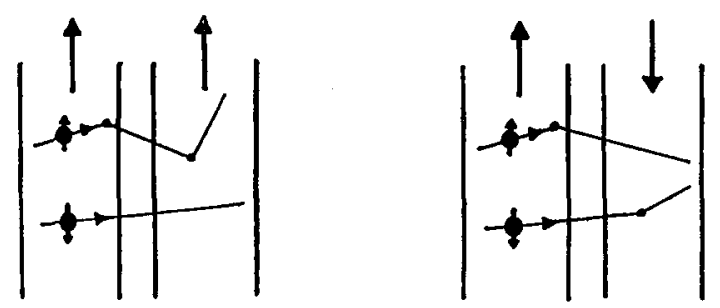

Fig. 2. Schematic electron conduction in layered structures with strong spin-asymmetry in scattering probabilities from impurities inside magnetic films.

whereas the spin-majority electrons are scattered very strongly ("slow electrons"). In the parallel case the electronic charge is carried mainly by "fast electrons". In the antiparallel configuration the electrons of a given spin are spin-minority in one magnetic film but spin-majority in the second one. Consequently, there are no "fast electrons" in this configuration and the resistance is much larger than that in the parallel configuration. This qualitative picture explains the origin of the negative MR (positive $\Delta R / R_{\uparrow \uparrow}$ ). However, a positive MR (negative $\Delta R / R_{\uparrow \uparrow}$ ) is also allowed. This can take place in asymmetrical structures when the spin-asymmetry factors in neighbouring ferromagnetic films are opposite in the sense that if the spin-minority electrons in a given film are "fast electrons", they are "slow electrons" in nearest ferromagnetic films. The positive MR can also occur in structures with spin-asymmetry factors for interface scattering at two successive interfaces respectively larger and smaller than 1.0.

The above qualitative picture neglects the spin-flip scattering processes which mix both channels, but it is easy to note that such processes reduce the GMR.

\subsection{Quasi-classical description}

Semiclassical theory of GMR is based on the Boltzmann kinetic equation with the magnetic driving term neglected. For each sublayer the kinetic equation can be written in the form

$$
\frac{\partial g_{\sigma}(z, v)}{\partial z}+\frac{g_{\sigma}(z, v)}{\tau_{\sigma} v_{z}}=\frac{e E}{m v_{z}} \frac{\partial f_{\sigma}^{0}(v)}{\partial v_{x}}
$$

where the axes $z$ and $x$ are normal to the films and along the electric driving field $E$, respectively, whereas $g_{\sigma}(z, v)$ is the deviation of the electron distribution function from the equilibrium Fermi-Dirac distribution $f_{\sigma}^{0}(v)$ :

$$
g_{\sigma}(z, v)=f_{\sigma}(z, v)-f_{\sigma}^{0}(v)
$$

In general, $f_{\sigma}^{0}(v)$ is spin-dependent due to spin-dependent effective electron potential $U_{\sigma}$ in the ferromagnetic films. In Eq. (2) $e$ is the electron charge $(e>0)$ and $m$ is the electron effective mass assumed to be independent of the electron spin. In a general case we assume $\tau_{\uparrow} \neq \tau_{\downarrow}$ and $U_{\uparrow} \neq U_{\downarrow}$ in the ferromagnetic layers and $\tau_{\uparrow}=\tau_{\downarrow}$ and $U_{\uparrow}=U_{\downarrow}$ in the nonmagnetic films. Equation (2) can be easily solved analytically for each sublayer. Its general solution takes the form

$$
g_{\sigma}^{ \pm}(z, v)=\frac{e \tau_{\sigma} E}{m_{\sigma}} \frac{\partial f_{\sigma}^{0}(v)}{\partial v_{x}}\left[1+F_{\sigma}^{ \pm}(v) \exp \left(\frac{ \pm z}{\tau_{\sigma}\left|v_{z}\right|}\right)\right]
$$


where "+" and "-" correspond to $v_{z}>0$ and $v_{z}<0$, respectively and the functions $F_{\sigma}^{ \pm}(v)$ can be determined from appropriate boundary conditions. In the simplest approximation [4, 8-10] one can write the boundary conditions in the Fuchs-Sondheimer form

$$
\begin{aligned}
& g_{\sigma}^{-}\left(z=0^{-}\right)=T_{\sigma} g_{\sigma}^{-}\left(z=0^{+}\right)+R_{\sigma} g_{\sigma}^{+}\left(z=0^{-}\right), \\
& g_{\sigma}^{+}\left(z=0^{+}\right)=T_{\sigma} g_{\sigma}^{+}\left(z=0^{-}\right)+R_{\sigma} g_{\sigma}^{-}\left(z=0^{+}\right)
\end{aligned}
$$

for an interface at $z=0$. The parameters $T_{\sigma}$ and $R_{\sigma}$ are the spin-dependent coefficients of specular transmission and reflection, whereas $D_{\sigma}=1-T_{\sigma}-R_{\sigma}$ is the probability of a diffusive scattering at the interface. The factor $N_{\mathrm{s}}$ can be then defined as $N_{\mathrm{s}}=D_{-} / D_{+}$. The boundary conditions for outer surfaces can be obtained from Eqs. (5a) and (5b) by assuming $T_{\sigma}=0$ and neglecting the distribution function outside the structure. In the above approximation the transmission and reflection coefficients are constant, i.e., independent of the angle of incidence. In more realistic considerations this dependence has to be taken into account [11], particularly when one wants to analyse the influence of the potential $U_{\sigma}$. Having found $g_{\sigma}(z, v)$ in each sublayer one can find the current density $j_{\sigma}(z)$ inside each film (per unit length along the axis $y$ ) from the formula

$$
j_{\sigma}(z)=-e\left(\frac{m}{h}\right)^{3} \int v_{x} g_{\sigma}(z, v) \mathrm{d}^{3} v .
$$

By integrating over $z$ and summing over all sublayers one finds the total current flowing along the electric field, which allows to find the resistance of the structure for both parallel and antiparallel configurations and, consequently, the relative resistance change $\Delta R / R_{\uparrow \uparrow}$.

Such an approach has been extensively used for numerical calculations of negative MR in sandwiches, multilayers as well as in infinite superlattices [4, 8-11]. All basic features of the effect have been reproduced with relatively good agreement with the experimental data. However, there are too many parameters which determine the value of GMR, therefore detailed comparison is rather difficult. What follows from numerical calculations is that spin-asymmetry in bulk and/or interface scattering probability is required to generate GMR. Spin-dependent effective potentials in the magnetic films are not sufficient for GMR to occur.

Although all theoretical (and experimental) results published up to now concern negative MR (positive $\Delta R / R_{\uparrow \uparrow}$ ), the method can be also used for the case of positive MR (negative $\Delta R / R_{\uparrow \uparrow}$ ). Exemplary results for the case of an asymmetrical sandwich are shown in Fig. 3, where the factor $\Delta R / R_{\uparrow \uparrow}$ is shown vs. mean free path in nonmagnetic spacer. Only the interface contribution to GMR is taken there into account. For the symmetrical case (equal spin-asymmetry factors at both interfaces, $N_{\mathrm{s}}=N_{\mathrm{s}}^{\prime}$ ) the ratio $\Delta R / R_{\uparrow \uparrow}$ is positive (negative MR). In the asymmetrical case, $N_{\mathrm{s}}=1 / N_{\mathrm{s}}^{\prime}$, this ratio is of opposite sign (positive MR).

In the semiclassical approach described above the electron scattering by impurities inside the films and the scattering by interface roughness are not taken into account on equal footing and the role of interface scattering is rather underestimated. This inconsistency can be overcome, at least to some extent, by 


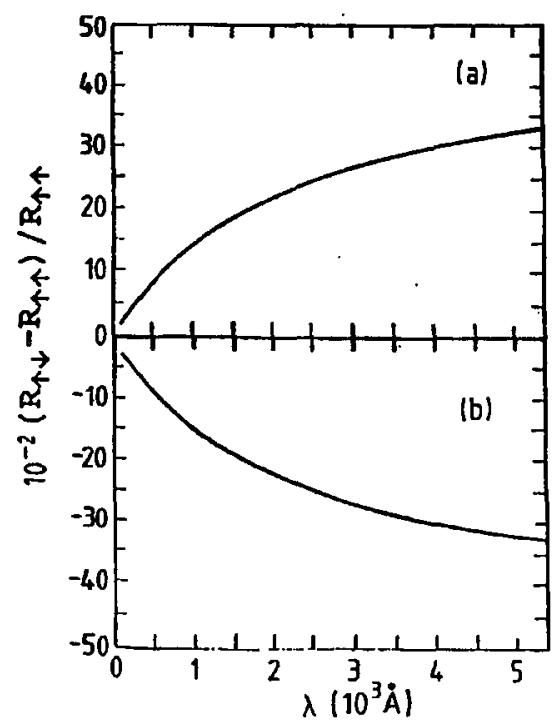

Fig. 3. Relative resistance change vs. mean free path in the nonmagnetic spacer in the case of negative (a) and positive (b) MR. The curves are calculated for a sandwich structure with $d_{\mathrm{m}}=10 \mathrm{~nm}, d_{\mathrm{n}}=1 \mathrm{~nm}, N_{\mathrm{b}}=1$ and specularity factor for outer surfaces $p=0.9$. Other parameters are $N_{\mathrm{s}}=N_{\mathrm{s}}^{\prime}=6, D_{-}=D_{-}^{\prime}=0.5$ (a) and $N_{\mathrm{s}}=1 / N_{\mathrm{s}}^{\prime}=6$, $D_{-}=D_{+}^{\prime}=0.5(\mathrm{~b})$. The parameters describing electronic properties of the nonmagnetic spacer are the same as those for magnetic films.

introducing a transition layer at the interface with a higher impurity concentration and with its own spin asymmetry factor. In that case the diffusive scattering is ruled out from the boundary conditions.

The above description applies to situations with no mixing between two spin channels. However, the spin-flip scattering processes can be included into the formalism and, as one might expect, they lead to some reduction of the GMR.

\subsection{Quantum model}

Quantum description of GMR is based on the Kubo approach formulated in real [12-15] or reciprocal [16] space. Within the real space Kubo formalism and for independent spin-channels the global in-plane conductivity $g_{\| \sigma}$ is given by the formula

$$
\begin{aligned}
g_{\| \sigma}= & \frac{1}{L} \int \mathrm{d} z \int \mathrm{d} z^{\prime} g_{x x}^{\sigma}\left(z, z^{\prime}\right)=-\frac{1}{4 L}\left(\frac{e}{m}\right)^{2} \frac{\hbar}{(2 \pi)^{2}} \int \mathrm{d} q \int \mathrm{d} z \int \mathrm{d} z^{\prime} q^{3} \\
& \times\left[G_{q \sigma}^{\mathrm{R}}\left(z, z^{\prime}\right)-G_{q \sigma}^{\mathrm{A}}\left(z, z^{\prime}\right)\right]\left[G_{q \sigma}^{\mathrm{R}}\left(z^{\prime}, z\right)-G_{q \sigma}^{\mathrm{A}}\left(z^{\prime}, z\right)\right],
\end{aligned}
$$

where $L$ is the total thickness of the structure. The retarded and advanced Green functions have been written here in a mixed representation and are taken at the Fermi energy $E_{F}$. 
The impurity averaged Green function $G_{q \sigma}^{\mathrm{R}}\left(z, z^{\prime}\right)$ fulfils the following equation:

$$
\frac{\hbar^{2}}{2 m} \frac{\mathrm{d}^{2}}{\mathrm{~d} z^{2}}+\left[E_{\mathrm{F}}-\frac{\hbar^{2} q^{2}}{2 m}-U_{\sigma}(z)+\mathrm{i} \frac{\hbar}{2 \tau_{\sigma}(z)}\right] G_{q \sigma}^{\mathrm{R}}\left(z, z^{\prime}\right)=\hbar \delta\left(z-z^{\prime}\right),
$$

where $U_{\sigma}(z)$ is a stepwise function describing the effective spin-dependent electron potential across the whole sample. The $z$-dependent relaxation time is given by the imaginary part of the corresponding self-energy $\Sigma(z)$ :

$$
\frac{1}{2 \tau_{\sigma}(z)}=-\operatorname{Im} \Sigma_{\sigma}(z)
$$

and the self-energy is determined by the impurity potential, impurity concentration and the unperturbed Green function of the problem. All the above three factors contribute to the $z$-dependence of the self-energy. In the following we use the approximation according to which $\tau_{\sigma}(z)$ is constant across each sublayer but may be different in different sublayers. This approximation simplifies Eq. (8) and practically enables finding its analytical solutions.

To find GMR one has then to calculate first the appropriate Green functions $G_{q \sigma}^{\mathrm{R}}\left(z, z^{\prime}\right)$ from Eq. (8) and then $G_{q \sigma}^{\mathrm{A}}\left(z, z^{\prime}\right)$ from the relation $G_{q \sigma}^{\mathrm{A}}\left(z, z^{\prime}\right)=$ $G_{q \sigma}^{\mathrm{R}^{*}}\left(z^{\prime}, z\right)$. This has to be done for both parallel and antiparallel configurations. Finally, one has to perform integrations indicated in Eq. (7). Consider for instance a bilayer consisting of two identical ferromagnetic films of thickness $d_{\mathfrak{m}}$ separated by an ultrathin spacer the thickness of which can be neglected. To calculate $G^{\mathrm{R}}\left(z, z^{\prime}\right)$ we will use the outgoing boundary conditions, which exclude the quantum size effects imposed by external confining potential. The size effects can be taken into

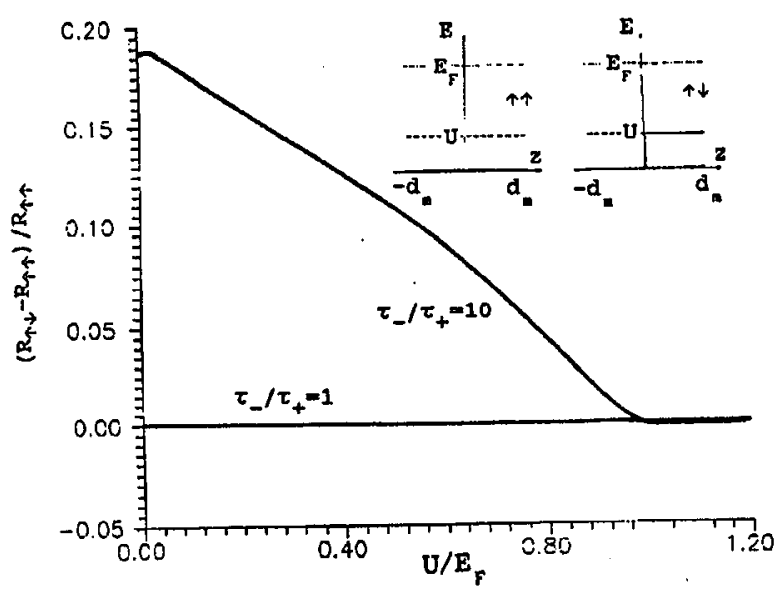

Fig. 4. Relative change of the resistance as a function of $U / E_{\mathrm{F}}$ for $d_{\mathrm{m}}=20 \mathrm{~nm}$, $E_{F}=3 \mathrm{eV}, \lambda_{+}=6 \mathrm{~nm}$ and $N_{\mathrm{b}}$ as indicated. The inset shows schematically the spin-dependent potential profiles in parallel and antiparallel configurations and for both spin directions. 
account by assuming hard wall boundary conditions [15]. The integrations over $z$ and $z^{\prime}$ can be easily performed analytically whereas the integrals over $q$ have to be calculated numerically. Some results are shown in Fig. 4, where the relative change of the resistances, $\left(R_{\uparrow \downarrow}-R_{\uparrow \uparrow}\right) / R_{\uparrow \uparrow}$ vs. $U / E_{\mathrm{F}}$ is presented for $N_{\mathrm{b}}=\tau_{-} / \tau_{+}=10$ and $N_{\mathbf{b}}=1$, where $U$ is the splitting factor of the electron bands as shown schematically in the inset in Fig. 4. The GMR decreases with increasing $U$ and vanishes for $U \Rightarrow E_{\mathrm{F}}$. For $\tau_{-}=\tau_{+}$there is no GMR. Thus, a spin asymmetry in relaxation times is required to generate GMR.

The above results describe so-called bulk contribution to GMR. The interface roughness was not taken into account. However, it can be easily included into the formalism by assuming a transition layer at the interface with a higher impurity concentration and with another spin-asymmetry factor.

\section{CPP geometry}

\subsection{Macroscopic limit}

Consider now electric current flowing perpendicularly to a layered structure. For simplicity we restrict considerations to periodic superlattices. Let us assume first the limit of infinite spin-diffusion length. It is clear that in the case of antiparallel orientation both spin currents are equal, $j_{\uparrow}=j_{\downarrow}$. For parallel alignment $j_{\uparrow} \neq j_{\downarrow}$, in general. In the macroscopic limit the resistance of the structure is equal to the resistance of the resistor network shown schematically in Fig. 5 for parallel and antiparallel alignment. In the CPP case one has to include additionally some boundary resistances which exist even in the limit of ideal (flat) interfaces. It is easy to calculate the relative change $\Delta R / R_{\uparrow \uparrow}$ of the resistance, which is equal to

$$
\Delta R / R_{\uparrow \uparrow}=\frac{\left[\left(\rho_{+}-\rho_{-}\right) d_{\mathrm{m}}+2\left(r_{+}-r_{-}\right)\right]^{2}}{4\left(\rho_{+} d_{\mathrm{m}}+\rho_{\mathrm{n}} d_{\mathrm{n}}+2 r_{+}\right)\left(2 \rho_{-} d_{\mathrm{m}}+\rho_{\mathrm{n}} d_{\mathrm{n}}+2 r_{-}\right)},
$$

where $\rho_{+}$and $\rho_{-}$are the resistivities of the magnetic films for spin-majority and

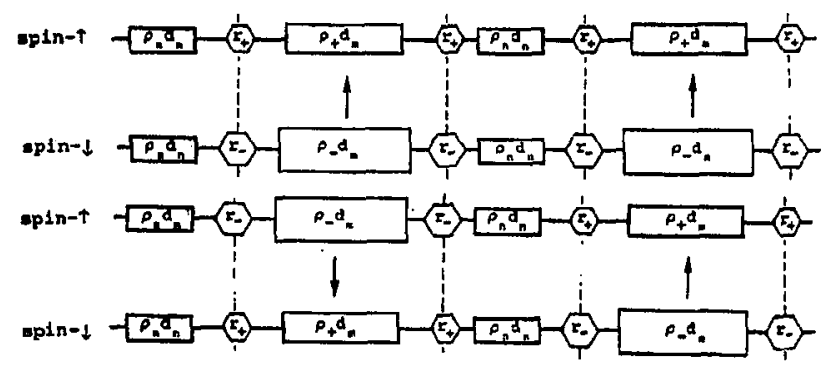

Fig. 5. The resistor network model for parallel and antiparallel configurations in the CPP geometry. 
spin-minority channels, $\rho_{\mathrm{n}}$ is the resistivity of the nonmagnetic spacer (per one spin channel) and $r_{+}$and $r_{-}$are the boundary resistances of a unit square for spin-majority and spin-minority electrons. If $\rho_{+} \neq \rho_{-}$and/or $r_{+} \neq r_{-}$, then the GMR does not vanish in the macroscopic limit for the CPP configuration.

Consider the case when $r_{+}=r_{-}$. The MR is nonvanishing if there is any spin-asymmetry in bulk resistivities of the ferromagnetic metal. Taking into account simple Drude formula for resistivity, $\rho_{\sigma}=m / n_{\sigma} e^{2} \tau_{\sigma}$, one can see that there are two factors which contribute to GMR. The first is the spin-asymmetry in relaxation times (as in the case of CIP geometry) and the second one is the spin-asymmetry in electron concentrations. The latter comes from spin splitting of the electron bands. Thus, the spin-dependent effective potential $U_{\sigma}$ can generate GMR even if $\tau_{+}=\tau_{-}$. The relevant parameter for the CPP geometry is then the spin-asymmetry factor for bulk resistivities, which takes into account both factors mentioned above. If $\rho_{+}=\rho_{-}$then the GMR can still occur if there is a spin-asymmetry in the boundary resistances, i.e. if $r_{+} \neq r_{-}$.

The above resistor network model was used successfully to account for recent experimental data obtained on $\mathrm{Co} / \mathrm{Cu}$ and $\mathrm{Co} / \mathrm{Ag}$ multilayers [5]. The appropriate analysis showed a relatively large spin asymmetry factor for the interface resistances, $r_{-} / r_{+} \approx 12$ in $\mathrm{Ag} / \mathrm{Co}$ for example, and a definitely smaller corresponding factor for the bulk resistivities of $\mathrm{Co}, \rho_{-} / \rho_{+} \approx 2.9$. The reason why those factors differ so much is not clear $[13,17]$. However, some conclusions can be obtained from analysis of the boundary resistance in a simple model situation shown in the inset in Fig. 6, when there is a potential step of height $U$ at the interface and some impurities are located at the interface with the areal concentration $n$ (for clarity we will omit the spin index in this paragraph). To calculate $r$ it is convenient to apply the Landauer formula. On averaging over the impurity distribution one finds

$$
r=\frac{\pi h}{4 e^{2}} \frac{1}{k_{\mathrm{F}}^{2}} \frac{1+J_{2}+x_{\mathrm{c}}-4 J_{1}\left(1-x_{\mathrm{c}}^{2}\right)^{-1 / 2}}{J_{3}},
$$

where $k_{\mathrm{F}}$ is the Fermi wave vector $\left(E_{\mathrm{F}}=\hbar^{2} k_{\mathrm{F}}^{2} / 2 m\right)$, and $J_{1}, J_{2}$ and $J_{3}$ are defined as follows:

$$
\begin{aligned}
& J_{1}=\int_{x_{\mathrm{c}}}^{1} \mathrm{~d} x x^{2} / f(x), \\
& J_{2}=\int_{x_{\mathrm{c}}}^{1} \mathrm{~d} x\left[2 x^{2}-x_{\mathrm{c}}^{2}-2 x\left(x^{2}-x_{\mathrm{c}}^{2}\right)^{1 / 2}\right] / f(x), \\
& J_{3}=\int_{x_{\mathrm{c}}}^{1} \mathrm{~d} x x^{2}\left(x^{2}-x_{\mathrm{c}}^{2}\right)^{1 / 2} / f(x),
\end{aligned}
$$

where

$$
f(x)=2 x^{2}-x_{\mathrm{c}}^{2}+2 x\left(x^{2}-x_{\mathrm{c}}^{2}\right)^{1 / 2}+\frac{\Gamma}{4 \pi}\left\{x+\left[\left(x^{2}-x_{\mathrm{c}}^{2}\right)\left(1-x_{\mathrm{c}}^{2}\right)\right]^{1 / 2}\right\}
$$

and $\Gamma$ and $x_{\mathrm{c}}$ are defined as

$$
\Gamma=\left(2 m / \hbar^{2}\right)^{2} V^{2} n,
$$




$$
x_{\mathrm{c}}=\left(U / E_{\mathrm{F}}\right)^{1 / 2} .
$$

Results of appropriate numerical calculations are shown in Fig. 6 for several values of the parameter $\Gamma$. For $\Gamma=0$ (perfect step) the resistance $r$ increases from $r=0$ at $U=0$ to about $5.0 \mathrm{f} \Omega \mathrm{m}^{2}$ ( $\mathrm{f} \equiv$ femto $=10^{-15}$ ) at $U / E_{\mathrm{F}} \approx 0.7$. As $U$ approaches $E_{\mathrm{F}}$ the resistance $r$ increases to infinity, as one might expect.

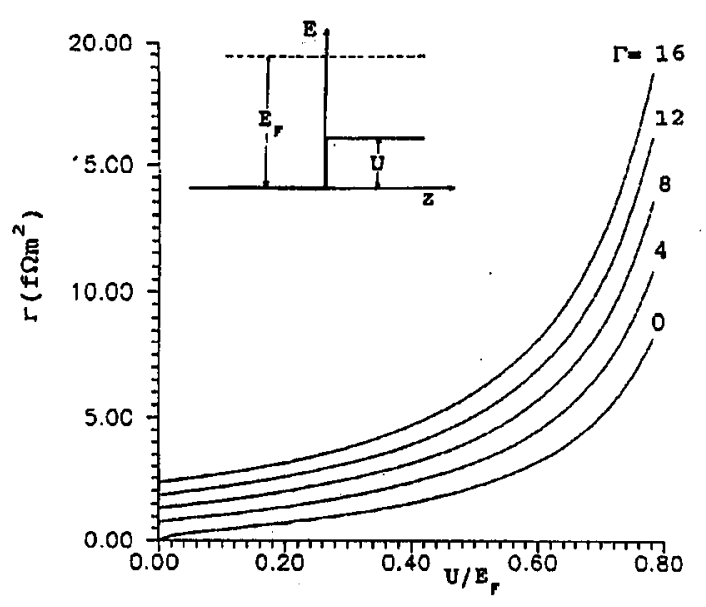

Fig. 6. Interface resistance $r$ of a unit square vs. $U / E_{F}$ for $q_{F}=1.3 \times 10^{-10} \mathrm{~m}^{-1}$ and for $\Gamma$ as indicated. The inset shows the geometry.

In a general case the potential step for majority electrons is different from that for minority electrons. Consequently, the resistances $r_{+}$and $r_{-}$are also different. Any value of the asymmetry factor $r_{-} / r_{+}$can be obtained. For $\Gamma>0$ the curves are shifted up. However, it is impossible to separate the contributions from scattering by impurities and the potential step on the basis of a vailable data.

One can now include easily the spin-flip scattering processes within the macroscopic limit. This has been done first by Valet and Fert [18]. When both spin-currents are mixed then only the total current is conserved across the structure. As was noticed first by Johnson and Silsbee [19] and van Son et al. [20] transport across an interface between ferromagnetic and nonmagnetic metals leads to spin accumulation at the interface. The accumulation layer extends on both sides of the interface up to the distance of the order of spin-diffusion length $l_{\sigma}^{\text {sf }}$, $l_{\sigma}^{\text {sf }}=\left(D_{\sigma} \tau_{\sigma}^{\mathrm{sf}}\right)^{1 / 2}$, with $D_{\sigma}$ being the diffusion constant $D_{\sigma}=v_{\mathrm{F}}^{\sigma} \lambda_{\sigma} / 3$. To describe both currents one introduces two electrochemical potentials $\mu_{\uparrow}$ and $\mu_{\downarrow}$ which are different, in general. The difference in the electrochemical potentials $\Delta \mu=\mu_{\uparrow}-\mu_{\downarrow}$ obeys the diffusion-type equation

$$
\frac{\partial^{2}}{\partial z^{2}}\left[\mu_{\uparrow}(z)-\mu_{\downarrow}(z)\right]=\frac{1}{l_{\mathrm{sf}}^{2}}\left[\mu_{\uparrow}(z)-\mu_{\downarrow}(z)\right]
$$

in which $l_{\mathrm{sf}}$ is an average of spin diffusion lengths $1 / l_{\mathrm{sf}}=1 / l_{+}^{\mathrm{sf}}+1 / l_{-}^{\mathrm{sf}}$. Additional 
equation follows from the $\mathrm{Ohm}$ law relating the current densities and the driving forces $(1 / e) \partial \mu / \partial z$ :

$$
\rho_{\sigma} j_{\sigma}(z)=\frac{1}{e} \frac{\partial \mu_{\sigma}}{\partial z}
$$

(we assume constant resistivity across each sublayer). The above two equations supplemented with the condition of the current conservation $\partial\left(j_{\uparrow}+j_{\downarrow}\right) / \partial z=0$ form a basic set of macroscopic equations which allow to find the chemical potentials, driving forces and current densities inside each constituting film. Those solutions have to be matched with appropriate boundary conditions

$$
e\left[\mu_{\sigma}\left(z_{0}^{+}\right)-\mu_{\sigma}\left(z_{0}^{-}\right)\right]=r_{\sigma} j_{\sigma}\left(z_{0}\right)
$$

for an interface at $z=z_{0}$. No spin-flip scattering at the interface is assumed here. This procedure allows to find resistance in both parallel and antiparallel configurations and finally the relative change of the resistance at the rotation. Appropriate formulae have been derived by Valet and Fert [18]. The general tendency is that spin-flip scattering processes lower the MR. When the sublayer thicknesses are much smaller than the spin-diffusion length, then the model reduces to the simple resistor network model discussed at the beginning of this section.

\subsection{Ballistic range}

Consider now the limit opposite to that described above, i.e. the ballistic transport across the superlattice. This type of transport in magnetic structures was analysed first by Bauer [21]. Consider a simple case when the spin majority electron band in the ferromagnetic metal matches well the conduction band in the nonmagnetic spacer. The appropriate potential profiles across the structure in both parallel and antiparallel configurations as well as for both spin directions are shown in Fig. 7. It is evident that the total number of quantum channels in

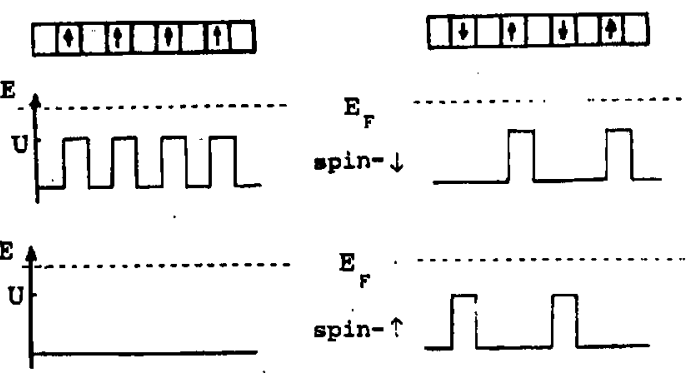

Fig. 7. Potential profiles for parallel and antiparallel orientations of the film magnetizations and for both spin directions.

the antiparallel configuration is lower than in the parallel one. According to the Landauer formula the conductance of a quantum wire (per spin) is

$$
G=\frac{e^{2}}{h} \operatorname{Tr} t^{+} t
$$


where $t$ is the matrix describing amplitudes of transition probabilities between different channels. In the ballistic range $\operatorname{Tr} t^{+} t \approx N$, where $N$ is the number of quantum channels and $N=S k_{\mathrm{F}}^{2} / 4 \pi$ ( $S$ is the cross-section of the wire). Taking this into account one finds the difference of conductances in parallel and antiparallel configurations

$$
\Delta G=G_{\mathrm{F}}-G_{\mathrm{AF}}=\frac{e^{2}}{h} \frac{S k_{\mathrm{F}}^{2}}{4 \pi}\left(1-\frac{E_{\mathrm{F}}-U}{E_{\mathrm{F}}}\right) .
$$

For $U=0$ there is no MR $(\Delta G=0)$, whereas for $U=E_{\mathrm{F}}$ the conductance in the AP configuration vanishes and $\Delta G=G_{F}$, which corresponds to infinite ratio $\Delta R / R_{\uparrow \uparrow}$ :

$$
\frac{\Delta R}{R_{\uparrow \uparrow}} \Rightarrow \infty \text { for } U \Rightarrow E_{\mathrm{F}}
$$

This behaviour is opposite to that in the CIP geometry where there is no effect when there is no spin-asymmetry in scattering probabilities, but even if it exists it vanishes as $U \Rightarrow E_{\mathrm{F}}$.

In the range between the ballistic and macroscopic limits the MR behaves similarly to the MR in the CIP geometry, therefore we will not analyse it in detail.

\section{Summary}

Current understanding of the GMR is based on the spin-dependent probabilities of electron scattering either from interface roughness or from impurities distributed inside the magnetic films. In the CPP geometry the periodic spin-dependent effective electron potential $U_{\sigma}$ may also play an important role and can generate GMR even when the scattering probability is spin-independent. Detailed comparison between the theory and experiment is difficult due to the fact that all theoretical models, even the simplest ones, contain parameters which are unknown. This is particularly true for parameters describing the interface scattering. Detailed calculations of the electronic structure might be helpful and could allow to determine those parameters from the microscopic calculations.

\section{References}

[1] M.N. Baibich, J.M. Broto, A. Fert, F. Nguyen van Dau, F. Petroff, P. Etienne, G. Creuzet, A. Friedrich, J. Chazelas, Phys. Rev. Lett. 61, 2472 (1988).

[2] G. Binasch, P. Grunberg, F. Saurenbach, W. Zinn, Phys. Rev. B 39, 4824 (1989).

[3] S.S.P. Parkin, N. More, K.P. Roche, Phys. Rev. Lett. 64, 2304 (1990).

[4] J. Barnaś, A. Fuss, R.E. Camley, P. Grunberg, W. Zinn, Phys, Rev. B 42, 8110 (1990).

[5] W.P. Pratt Jr., S.F. Lee, J.M. Slaughter, P.A. Schroeder, J. Bass, Phys. Rev. Lett. 66, 3060 (1991); S.F. Lee, W.P. Pratt Jr., R. Loloee, P.A. Schroeder, J. Bass, Phys. Rev. B 46, 548 (1992).

[6] M.A.M. Gijs, S.K.J. Lenczowski, J.B. Giesbers, to be published.

[7] I.A. Campbell, A. Fert, in: Ferromagnetic Materials, Ed. E.P. Wohlfarth, North-Holland, Amsterdam 1982, p. 769. 
[8] R.E. Camley, J. Barnaś, Phys. Rev. Lett. 63, 664 (1989).

[9] B. Dieny, J. Phys., Condens. Matter 4, 8009 (1992).

[10] A. Barthelemy, A. Fert, Phys. Rev. B 43, 13124 (1991).

[11] R.Q. Hood, L.M. Falicov, Phys. Rev. B 46, 8287 (1992).

[12] A. Vedyayev, B. Dieny, N. Ryzhanova, Europhys. Lett. 19, 329 (1992).

[13] J. Barnaś, A. Fert, to be published.

[14] H.E. Camblong, P.M. Levy, Phys. Rev. Lett. 69, 2835 (1992).

[15] B. Bułka, J. Barnaś, proceedings of this conference, Part II.

[16] P.M. Levy, S. Zhang, A. Fert, Phys. Rev. Lett.65, 1643 (1990); S. Zhang, P.M. Levy, A. Fert, Phys. Rev. B 45, 8689 (1992).

[17] J. Barnas, A. Fert, submitted to Phys. Rev. B.

[18] T. Valet, A. Fert, to be published.

[19] M. Johnson, R.H. Silsbee, Phys. Rev. B 35, 4959 (1987).

[20] P.C. van Son, H. van Kempen, P. Wyder, Phys. Rev. Lett. 58, 2271 (1987).

[21] G.E.W. Bauer, Phys. Rev. Lett. 69, 1676 (1992). 